\title{
Konstruktions- und Montageprinzipien des Fixateur externe
}

\author{
Dankward Höntzsch
}

\section{Zusammenfassung}

Die externe Fixation ist eine der Hauptsäulen der operativen Frakturbehandlung. Diese Osteosyntheseform ermöglicht die lokale DamageControl-Chirurgie für Frakturen mit ausgedehnten Weichteilschäden und für polytraumatisierte Patienten. Darüber hinaus ist sowohl die Korrektur von Deformitäten als auch der Knochensegmenttransport möglich. Arthrodesen und die septische Chirurgie profitieren von der externen Fixation. Um diese Aufgaben zu erfüllen, stehen der externen Fixation reichhaltige Variationen zur Verfügung. Der Fixateur externe ist aus 3 Elementen aufgebaut: Fassen der Knochenfragmente (Pins und/oder Drähte) mit extern gelegenen Enden, den Längsträgern und Ringen und den Verbindungselementen. Die Grundzüge der externen Fixation lassen sich mit wenigen Konstruktions- und Montageprinzipien beschreiben. Dabei sollte die Form, d.h. die Konstruktion und die Montage der Funktion folgen („Form follows function“). Wenn diese Prinzipien eingehalten werden, kann die externe Fixation erfolgreich eingesetzt werden.

\section{Principles of External Fixator Design and Assembly}

External fixation is one of the mainstays of surgical fracture treatment. This method of osteosynthesis facilitates local damage control surgery for fractures with extensive soft tissue injury and in polytraumatised patients. In addition, correction of deformities and bone segment transport are possible. Arthrodesis and septic surgery also benefit from external fixation. An extensive range of fixators and methods is available to meet these various needs. An external fixator consists of 3 elements: the first part for securing the bone fragments (pins and/or wires with extracorporeal ends), longitudinal rods and rings, and the connectors. The main features of external fixation can be described in terms of a few design and assembly principles. It is important that "form follows function", i.e., design and assembly are modified to the function. If these principles are respected, external fixation can be applied with success.

\section{Einleitung}

„Form follows function“ - die Form, d.h. die Konstruktion und Montage folgen der Funktion.

Mit den verschiedenen Konstruktionsund Montageprinzipien des Fixateur externe kann dies formvollendet verwirklicht werden. Der Fixateur ist nicht das „schwarze Schaf“ oder der „verlorene

OP-JOURNAL 2008; 24: 4-9

(c) Georg Thieme Verlag KG Stuttgart • New York DOI 10.1055/s-2008-1038427

Sohn“ in der Familie der Osteosyntheseverfahren. Der Fixateur wird differenziert eingesetzt und montiert und ist eine der wichtigsten Säulen im Spektrum der verschiedenen Osteosyntheseverfahren.

\section{Warum externe Fixation?}

Die externe Fixation findet vor allem immer dann Anwendung, wenn die lokalen und/oder allgemeinen Verhältnisse beim Patienten schwierig sind und wenn äußere Bedingungen die externe Fixation nahe legen $[4,5,8]$.
Vorteile der externen Fixation:

- Geringe zusätzliche Verletzung der Weichteile und Knochen

- Minimales zusätzliches Weichteiltrauma

- Zeitsparende Anwendung in der Notfallsituation

- Stabilisation von offenen und kontaminierten Frakturen

- Sekundäre Frakturreposition oder Modifikation der Stabilität der Fixation ohne weitere operative Eingriffe

- Gute Alternative bei Infektrisiko

- Geringere chirurgische Erfahrungen erforderlich als bei internen Osteosyntheseverfahren

- Segmenttransport, Verlängerung und Deformitätenkorrektur möglich

- Bei Primärversorgung Ausbehandlung im Fixateur oder Verfahrenswechsel möglich

- Bei Infektsituation Verfahrenswechsel von der inneren Osteosynthese zur externen Fixation möglich

Die lange Liste der möglichen Indikationen soll einleitend nur tabellarisch dargestellt werden.

Übersicht über die Indikationsmöglichkeiten der externen Fixation:

- Einfache und/oder komplexe Frakturen mit offenem oder geschlossenem Weichteilschaden

- Polytraumatisierte Patienten

- Bei Schaftfrakturen

- Bei Gelenkfrakturen

- Beckenfrakturen

- HWS-Verletzungen mit Halo-Fixateur

- Frakturen mit Knochen- und Weichteilverlust

- Fixations- und Transportmittel bei Kallusdistraktion für Verlängerung und Segmenttransport

- Als Distraktor und/oder als modulares Repositionsinstrument (siehe 3-RohrModulartechnik)

\section{Elemente der externen Fixation}

Das Prinzip der externen Fixation besteht darin, dass die Knochenfragmente, welche reponiert und stabilisiert werden sollen von einseitig eingebrachten 


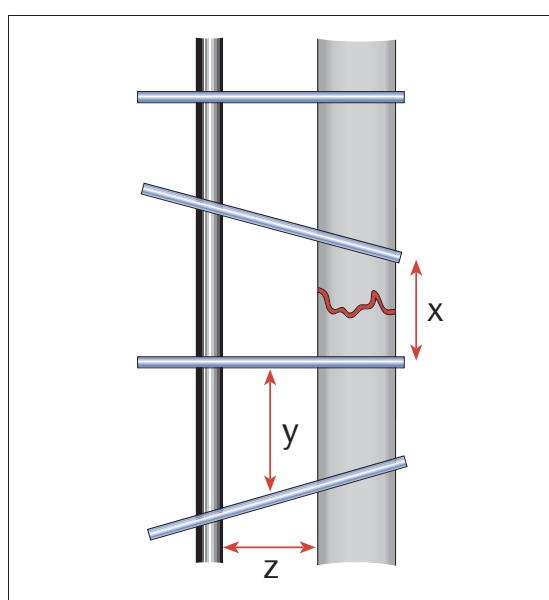

Abb. 1 Kriterien, welche die Steifigkeit und Stabilität der Fixateur-externe-Montage bestimmen.

Knochenschrauben (Schanz-Schrauben), von durchgehenden Stiften (SteinmannPins), von gespannten Drähten (bei Ringund Hybridsystemen) oder von außen wirkenden zangenartigen Greifern (Halo-Fixateur und Zangen- bzw. PinlessFixateur) gefasst werden. Die herausstehenden Enden werden dann mit verschiedenartigen äußeren Elementen gehalten.

Diese äußeren Fixationselemente können einfache Metallstäbe oder Rohre, röntgendurchlässige Kohlefaserstäbe, komplexe mehrgelenkige Stabilisationssysteme oder Gewindestangen beim Ringfixateur und Transportfixateuren sein.

Die Verbindung zwischen den Knochenfixationselementen und den externen Fixationselementen wird von Verbindungsbacken verschiedenster Art hergestellt.

Jedes dieser 3 Bauelemente der externen Fixation kann von einfach bis hochkomplex sei. Die grundsätzlichen Konstruktions- und Montageprinzipien werden davon aber nicht berührt.

Fixateur-externe-Elemente zur Knochenfixation:

- Unilaterale Knochenschrauben = Schanz-Schrauben

- Durchgehende Metallstife = Steinmann-Pins

- Gespannte Drähte = für Ring- und Hybridsysteme

- Zangen = Halo-Fixateur/Pinless oder Zangenfixateur

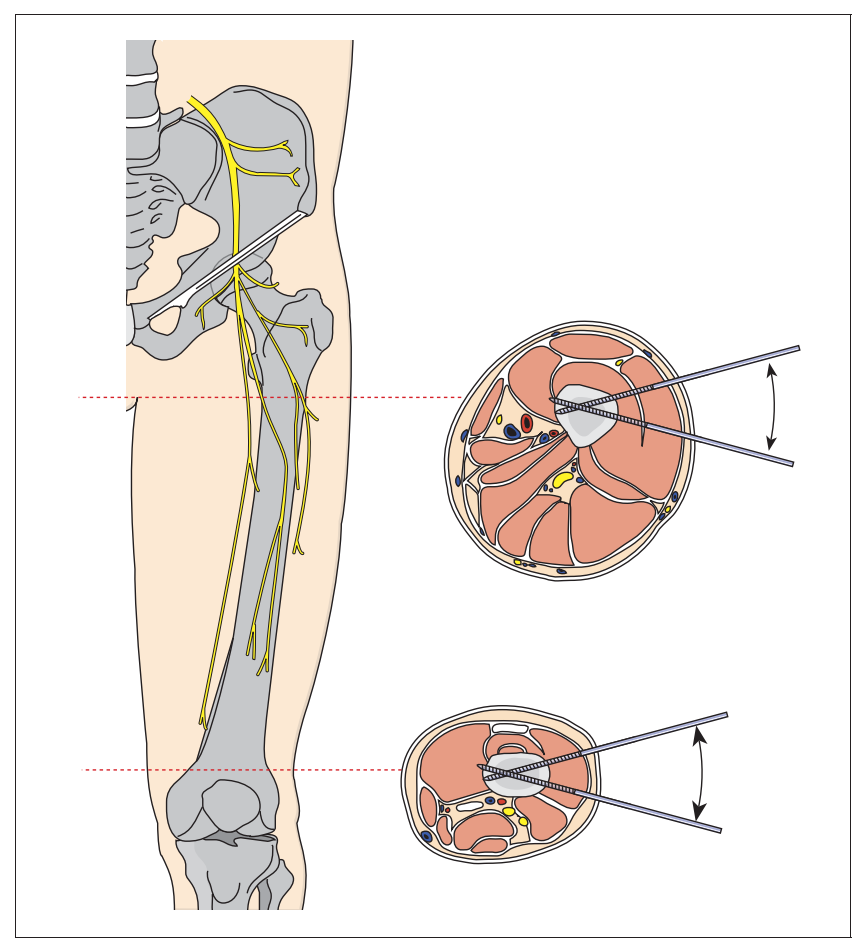

Abb. 2 Sichere Zonen am Oberschenkel (s. Seite 9).
Fixateur-externe-Elemente externe Längsträger:

- Metallstäbe/Rohre

- Kohlefaserstäbe

- Gewindestangen

- Unilaterale „Bodysysteme“ (z. B. MEFiSTO ${ }^{\circledR}$, ALF $^{\circledR}$, Heidelberger Ex FIX ${ }^{\circledR}$, Monotube ${ }^{\circledR}$ u. a.)

Fixateur-externe-Elemente -

Verbindungsbacken:

- Einfache Rohr/Stab- zu Pinbacken

- Ring/Drahtbacken zum Spannen der Drähte

- Rohr/Stab- zu Rohr/Stabbacken (Tube to Tube Clamps)

- Geschlossene Backen

- Seitlich offene Backen mit oder ohne Federsystemen

- Kombinationsbacken

- Mehrfach-, sog. Multipinbacken

- Sonderformen

- Backen aus Stahl, Titan oder Kunststoff (röntgendurchlässig)

\section{Biomechanische Montageprinzipien}

Der Fixateur externe sollte ausreichend Stabilität zum Aufrechterhalten der Reposition unter den verschiedenen Belastungsbedingungen gewährleisten [7]. Hierzu müssen die biomechanischen Prinzipien verstanden werden. Im Regelfall werden mindestens 2 Pins oder gespannte Drähte in jedes Fragment/ Segment eingebracht. Bei der Platzierung der Pins und/oder der Drähte sollte die Verletzungsregion des Knochens und vor allem der Weichteile berücksichtigt werden. Die Position der Pins sollte in den anatomisch sicheren Zonen liegen. Eine gute Montage berücksichtigt auch soweit wie möglich eine evtl. sekundäre innere Osteosynthese.

Die Steifigkeit und Stabilität der Fixateur-externe-Montage hängt von folgenden Faktoren ab: (Abb. 1) [3, 5, 7]

- Abstand der Pins/Schanz-Schrauben von der Fraktur (x): je näher, desto steifer

- Abstand der Pins/Schanz-Schrauben innerhalb eines Fragments (y): je gröBer der Abstand, umso steifer

- Abstand der longitudinalen Verbindungsstäbe vom Knochen (z): je näher, umso steifer

- Anzahl und Stärke der Längsträger

- Konfiguration der Montage

- Stärke und Material der Schrauben (d)

\section{Sichere Zonen (Abb. 2 und 3)}

Um Verletzungen von Nerven, Gefäßen, Sehnen und Muskeln zu vermeiden, müssen anatomische Kenntnisse vor allem projiziert auf den Querschnitt der Extremität berücksichtigt werden. Dies wird in speziell darauf abgestellten anatomischen Ausführungen dargestellt [1, 5]. Bei den transversen Drähten für die Ringfixateure und Hybridfixateure und bei Steinmann-Nägeln müssen die zugewandte Insertionsseite und die $\mathrm{Ge}$ - 

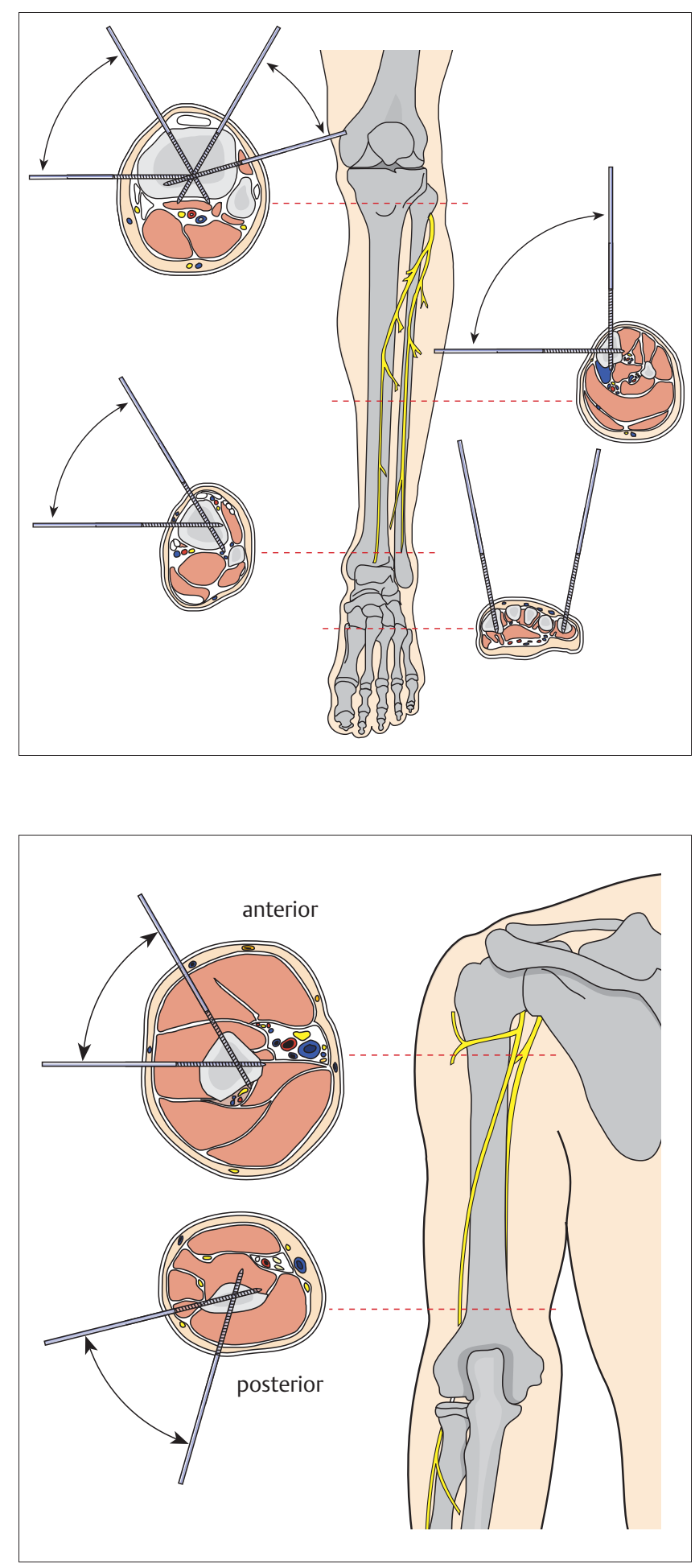

Abb. 3 Sichere Zonen am Unterschenkel (s. Seite 9).

Abb. 4 Sichere Zonen am Oberarm. genseite berücksichtigt werden (Abb. 2 bis 4).

Schanz-Schrauben, Steinmann-Pins und Drähte sollten das Muskel- und Sehnenspiel nicht stören. Es bewährt sich, während oder vor allem nach der Insertion und Stabilisierung die angrenzenden Gelenke durchzubewegen, um evtl. Kor- rekturen an der Haut- und Faszieninzision oder im Einzelfall eine Neuplatzierung vornehmen zu können. So kann das Grundprinzip „Form folgt Funktion“ konsequent umgesetzt werden.

Eine konsequent korrekte Platzierung reduziert die Infektrate für die Pins, fördert die Bewegungsmöglichkeiten der angrenzenden Gelenke, macht den Fixateur externe schmerzarm, erhöht den Tragekomfort und hilft, die Tragezeit des Fixateur externe zu verlängern. Dies ist besonders dann wichtig, wenn eine längere Implantationszeit für den Fixateur geplant ist. Besonders wichtig ist dies zum Beispiel beim Ausbehandeln im Fixateur und bei Verlängerung und Segmenttransport.

Ein besonderes Augenmerk möchte der Autor auf folgende Empfehlung legen [5]: Bei unilateralen Fixateur-externeMontagen an der Tibia ist es nicht notwendig, die Schanz-Schrauben in die besonders dicke anteriore Tibiakante einzubringen. Auch die mediale Tibiakortikalis hat eine ausreichende Dicke. Schanz-Schrauben werden ohnehin bikortikal verankert. Das meist schwierige Aufbohren der harten Kortikalis an der Tibiakante kann mit exzessiver Hitzeentwicklung und folgender Knochennekrose (später Ringsequester) einhergehen. $\mathrm{Zu}$ beachten ist zudem, dass die Spitze des Bohrers von der Tibiakante medial und lateral leicht abrutscht. Die Insertion auf der medialen Fläche ist wesentlich leichter. Im Bereich der distalen Tibia besteht anterior ein Risiko der Verletzung bzw. Irritation der Sehnen der Musculi tibialis anteriores und extensor digitorum. Der weit distal gelegene Pin an der Tibia hat bei "falscher" Platzierung die höchste Infektionsrate, weil dann das Sehnenspiel einen Reizzustand fördert. Die sicherere und bessere Zone ist hier die anterior-mediale Fläche der Tibia (Abb. 4).

\section{Montageprinzipien}

Grundsätzlich werden unilaterale Rahmensysteme, bilaterale Rahmensysteme und Ringsysteme unterschieden. Mischformen sind trianguläre Konstruktionen. Dies findet zum Beispiel am sprunggelenksüberbrückenden triangulären Fixateur Anwendung. Eine weitere Mischform ist der Hybridfixateur als $\mathrm{Mi}$ schung zwischen einem Ring oder Teilringsystem und einem unilateralen System [6].

Für unilaterale Rahmen werden SchanzSchrauben eingesetzt. Bilaterale Rahmensysteme verwenden SteinmannPins und Ringsysteme gespannte Drähte. Die Ring- oder partiellen Ringsysteme können zusätzlich zu den gespannten Drähten mit Schanz-Schrauben besetzt werden. Letzteres wird manchmal auch als Hybridanordnung beschrieben. 
Deshalb ist darauf zu achten, welche Definition von Hybridfixateur in einer Beschreibung gemeint ist!

\section{Rahmensysteme}

Unilateraler Rahmen

Der unilaterale Rahmen ist die am häufigsten verwendete Montageform, um eine frische diaphysäre Fraktur mit einem Fixateur externe zu stabilisieren.

Der Rahmen wird dabei in einer Ebene angebracht, anteromedial oder medial an der Tibia und lateral am Femur. Die Pins werden durch Stichinzisionen bikortikal verankert. Eine Längsverbindung (Stab oder Rohr) ist ausreichend, wenn eine stabil reponierte Fraktur mit gutem Knochenkontakt vorliegt. Bei instabilen Frakturen oder bei Knochenverlust werden zwei Längsverbindungen verwendet, entweder beide in einer Ebene oder in zwei unterschiedlichen Ebenen, die dann verbunden werden.

\section{Bilateraler Rahmen}

Dieser Rahmentyp wird im Allgemeinen für Kompressionsarthrodesen des Kniegelenkes oder des Sprunggelenkes verwendet. Ein Steinmann-Pin wird dabei bikortikal eingebracht und penetriert die Haut auf beiden Seiten. Diese Montage wird für die Therapie von Frakturen heute nicht mehr empfohlen.

\section{Gelenküberbrückung}

Die gelenküberbrückende externe Fixation ist immer dann inzidiert, wenn entweder der Patient (z. B. Polytrauma), die Extremität oder die Fraktur nicht endgültig primär versorgt werden kann oder soll.

Diese Art der Versorgung ist angezeigt, um gelenknahe Frakturen mit schwerwiegenden Weichteilschäden oder komplexe Gelenkfrakturen und Frakturdislokationen temporär zu stabilisieren. Die Stabilisierung erlaubt eine Erholung der Weichteile. Der Patient kann gut gepflegt werden, und eine präoperative Planung ist möglich.

\section{Modulare Repositions- und Fixations- techniken (Abb. 5) $[2,3,5,6]$}

Besonders die „Baukastensysteme“ des Fixateur externe erlauben eine modulare Technik. Hierbei ist die Position der Schanz-Schrauben frei. Das Prinzip der

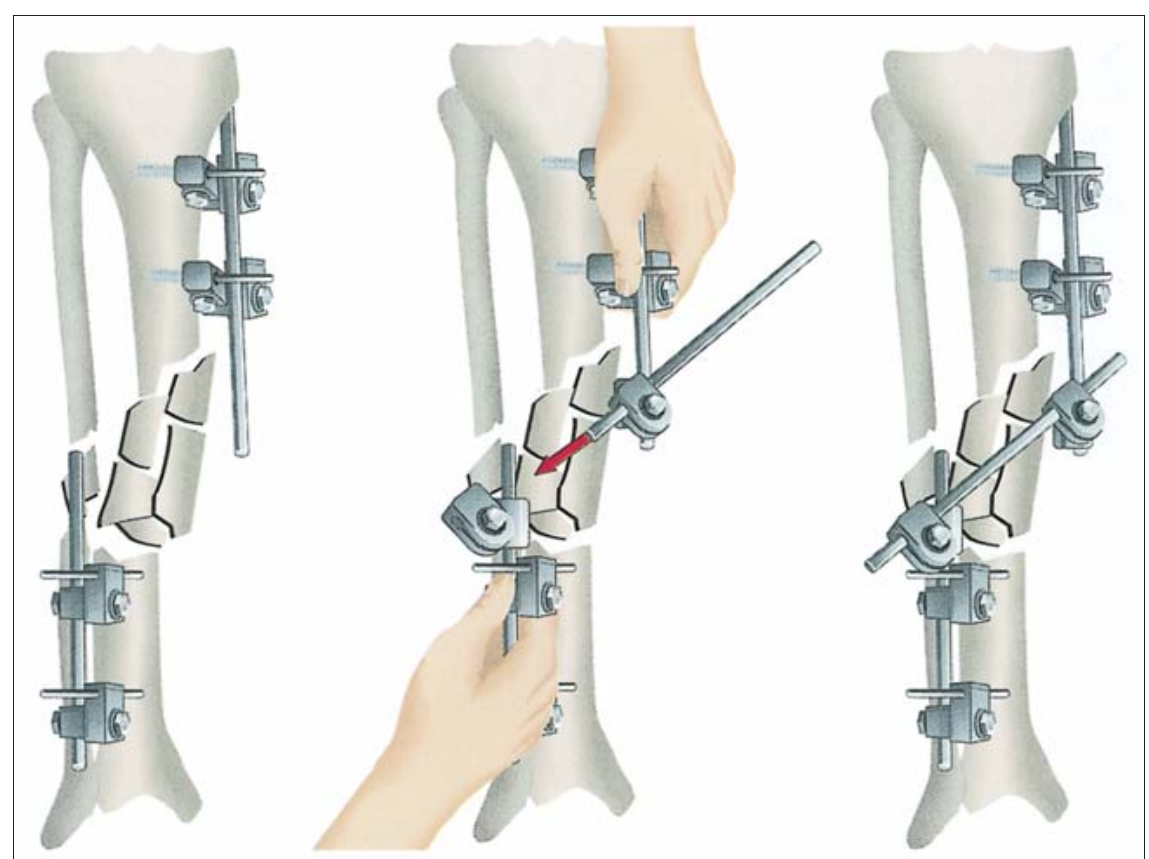

Abb. 5 Darstellung des Prinzips der Modulartechnik an der Tibia.

Modulartechnik ist, dass jedes Fragment mit einem Teil des endgültigen Rahmens besetzt wird. Mit und über diese Teilrahmen kann die Reposition erzielt werden. Die Teilrahmen werden dann mit einem modularen Zwischenelement verbunden und fixiert.

Dies kann am besten bildlich (z.B. am Unterschenkel) dargestellt werden. Die Modulartechnik kann mit dem Baukastensystem an jeder Schaftfraktur gelenknahen oder gelenküberbrückenden Montage angewendet werden (Abb. 5).

Stichwortartige Erklärung der 3-RohrModulartechik (Abb. 5):

Erster Schritt: Das körpernahe und das körperferne Hauptfragment werden jeweils mit 2 Schanz-Schrauben besetzt. Eintrittsort und -richtung der SchanzSchrauben ist absolut frei wählbar. Die Schrauben müssen in einer Ebene zueinander nicht parallel sein.

Der Ort kann nach anatomischer Vorgabe oder nach dem Verletzungsmuster ausgerichtet werden.

Zweiter Schritt: Diese jeweils 2 SchanzSchrauben pro Hauptfragment werden mit einem 11-mm-Rohr oder Stab und den entsprechenden Backen verbunden. Dieses Rohr/Stab muss zur Fraktur (Mitte) etwa 10-30 mm überstehen.

Dritter Schritt: Die Backen für diese Verbindung müssen fest angezogen werden!

Vierter Schritt: An den überstehenden Enden werden Rohr-Rohr-Backen aufgesetzt.
Fünfter Schritt: Durch die freien Löcher der Rohr-Rohr-Backen wird ein (genügend langes) drittes Rohr (deshalb 3-Rohr-Modulartechnik) geschoben. Solange reponiert wird, bleiben die Muttern dieser Rohr-Rohr-Backen offen! Dieser 5. Schritt, d. h. „drittes Rohr einschieben", kann auch nach dem nächsten Schritt 6, d.h. Reposition, ausgeführt werden!

Sechster Schritt: Nun wird die Fraktur reponiert. Die Teilrahmen (siehe Schritt zwei) können als „Griff“" benutzt werden, oder es werden temporäre Griffe angeschraubt.

Großer Vorteil: Haut, Weichteile, verletztes Gewebe müssen weder gedrückt noch gezogen werden. Repositionskräfte sind viel leichter übertragbar.

Siebenter Schritt: Die Reposition wird klinisch und röntgenologisch überprüft. Achter Schritt: Nach erfolgter Reposition werden die beiden Rohr-Rohr-Backen mit dem eingeschobenen dritten Rohr fest angezogen. Es empfiehlt sich schrittweises alternierendes Anziehen, damit die Backen noch etwas „spielen“ können und sich so selbst ausrichten.

Wichtiger Hinweis: Es ist vorteilhaft, wenn die Rohr-Rohr-Backen so gedreht werden, dass die Muttern einen „anschauen".

Neunter Schritt: Abschließende klinische und Röntgenkontrolle.

Zehnter Schritt: Durch Öffnen der zwei Rohr-Rohr-Backen kann die Reposition beliebig oft geändert werden. 


\section{Hybridfixateur (Abb. 6)}

Die Kombination zwischen unilateralem Fixateur am Schaft und einem Ringoder Teilringsystem, welches über gespannte Drähte ein meist gelenknahes kleineres Fragment hält, wird als Hybridfixateur bezeichnet.

Der Ring und der unilaterale Anteil des Fixateurs werden über entsprechende Backen unilateral bzw. in den meisten Fällen mit einer Dreieckskonstruktion lateral und medial und/oder ventral abgestützt. Mit diesem Hybridsystem können die Vorteile der unilateralen Montage am Schaft und die große Fähigkeit gespannter Draht-/Ringsysteme zum Halten kleiner gelenknaher Fragmente kombiniert werden.

\section{Ringsysteme (Abb. 7)}

Volle Ringsysteme haben den Vorteil, dass Belastungs- und Korrekturachse gleichermaßen durch das Zentrum des Ringsystems und der Längsachse des Knochens verlaufen. Verlängerung, Segmenttransport, einfache und besonders komplexe Achskorrekturen sind mit einem zirkulären Ringsystem möglich. Aber in vielen chirurgischen Schulen besonders im angloamerikanischen Raum werden Ringsysteme auch zur Frakturbehandlung verwendet. Dies wird im Kapitel „Ringfixateur für die Kallusdistraktion" ausführlich beschrieben.

\section{Prinzipien der Nachbehandlung und Dynamisierung}

Mit wenigen Ausnahmen (notfallmäßige Überbrückung) kann die Extremität von Beginn an teilbelastet werden. Während der Heilungsphase wird die Belastung langsam bis zur Vollbelastung gesteigert.

Beobachtungen über viele Jahre haben gezeigt, dass zusätzliche Dynamisierungselemente im Fixateur nicht notwendig sind. Teil- und Vollbelastung der Extremität unter externer Fixation sind die effektivste und beste Methode zur Dynamisierung.

\section{Halo-Fixateur}

Für die Halswirbelsäule gibt es eine besondere externe Fixation: Bei dem sogenannten Halo-Fixateur externe wird die Schädelkalotte mit einem schraubenbesetzten Ring gefasst (Halo). Dabei werden die Schraubenspitzen nur in die

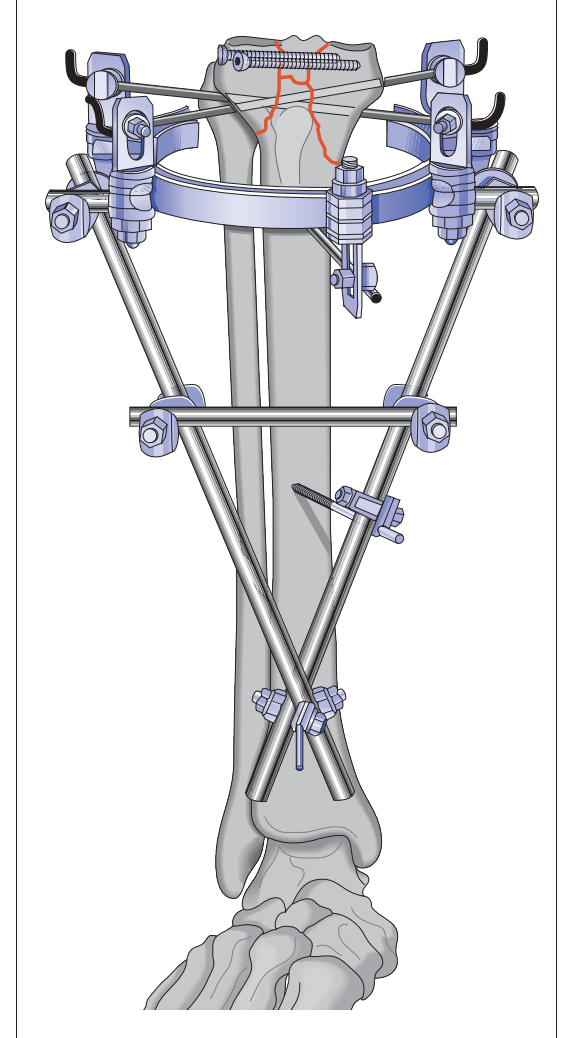

Abb. 6 Beispiel für einen Hybridfixateur.

äußere Kortikalis der Schädelkalotte eingedreht und der Kopf zentriert gehalten. Über ein äußeres Gestell wird dann gegenüber einer Kunststoffweste am Oberkörper und Schulter fixiert. Dadurch können Frakturen und Luxationen der Halswirbelsäule vorübergehend (bis zur notwendigen Operation) oder zum Teil auch abschließend behandelt werden.

\section{Verfahrenswechsel oder Ausbehandlung?}

Es gibt prinzipiell 3 Behandlungskonzepte:

1. definitive Behandlung mittels Fixateur externe bis zum Abschluss der Knochenbruchheilung

2. früher Verfahrenswechsel zur inneren Fixation

3. Verfahrenswechsel zu einer nichtoperativen Behandlung, z. B. Gips, Orthese etc.

Wenn ein Verfahrenswechsel zur inneren Fixation geplant ist, muss dieser so früh wie möglich durchgeführt werden, da ein späterer Umstieg zu einer höheren Komplikationsrate (Infektion) führen kann.

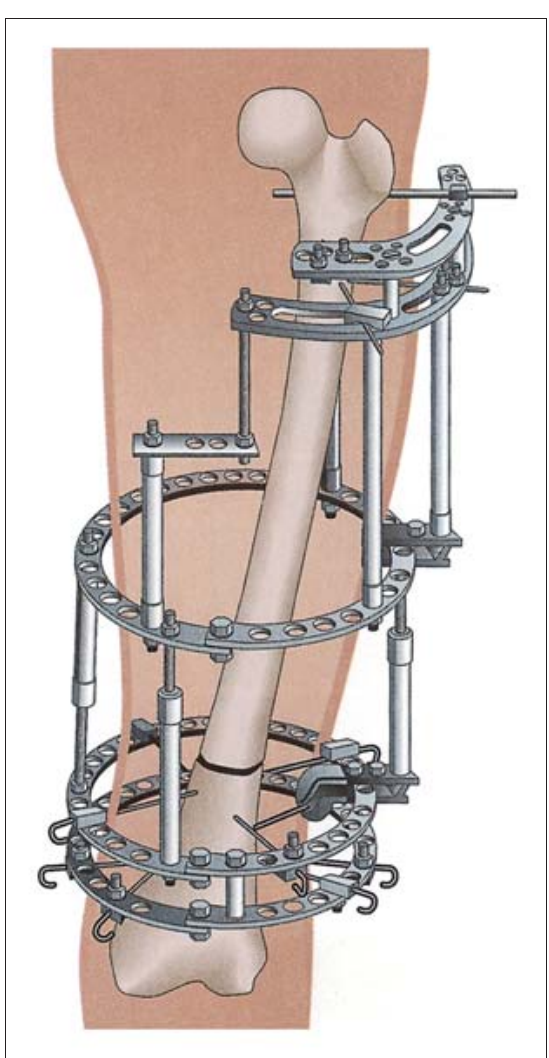

Abb. 7 Beispiel für einen Ringfixateur.

Wenn der Verfahrenswechsel erst nach Ablauf von 3-4 Wochen erfolgen kann oder bei Verdacht auf Pin-Infektion wird empfohlen, den Fixateur zu entfernen und die Extremität kurz andersweitig ruhigzustellen [5]. Sobald die Pin-Eintrittsstellen komplett abgeheilt sind, kann sekundär die innere Osteosynthese durchgeführt werden.

Die Notfallbehandlung mit einem Fixateur zur Stabilisierung der Extremität erlaubt die Erholung der Weichteile. Sobald die Weichteile es zulassen, kann die endgültige innere Osteosynthese erfolgen. Ein Verfahrenswechsel ist allerdings nicht zwingend, sofern der Fixateur stabil ist und es keine Komplikationszeichen gibt.

Der Fortschritt der Knochenheilung muss engmaschig überwacht werden. Bei fehlender Kallusbildung muss über eine alternative Behandlungsmethode nachgedacht werden.

\section{Schlussbemerkung}

In diesem Artikel sind Konstruktionsund Montageprinzipien beschrieben. Auf die Bedeutung der Modulartechnik wurde besonders eingegangen. 
Die Umsetzung der Konstruktions- und Montageprinzipien des Fixateur externe wird in diesem Heft im Einzelnen beschrieben: Komplikations- und Infektmanagement, Polytrauma, gelenküberbrückende Montagen, Bewegungsfixateure, kleine Fixateure, Ringfixateur, Kallusdistraktion, Korrekturoperation und Arthrodesen.

Hinweis zu den Abbildungen 2 und 3:

Die Abbildungen stammen aus Rüedi/ Buckley/Moran, AO Principles of Fracture Management (2007/ISBN-13: 9783-13-117442-0) mit freundlicher Genehmigung von AO Publishing, Copyright (C) 2007, by AO Publishing, Schweiz.

\section{Literatur}

${ }^{1}$ Faure C, Merloz PH. Zugänge für die Fixateur externe-Osteosynthese, Atlas anatomischer Querschnitte. Berlin, Heidelberg, New York: Springer Verlag, 1987

2 Fernandez Dell'Oca AA. External fixation using simple pin fixators. Injury 1992; 23 (Suppl 4): 1-54

${ }^{3}$ Fernandez A, Masliah R. Modular External Fixation in Emergency. Montevideo: Ingraf., 1991

${ }^{4}$ Giannoudis PV. Surgical priorities in damage control in polytrauma. J Bone Joint Surg [Br] 2003; 85: 478-483

${ }^{5}$ Höntzsch D, Bavonratanavech S. Fixateur externe. In: Ruedi T et al. (Eds). AO Principles of Fracture Treatment. AO Publishing. Stuttgart: Thieme Verlag, 2004

6 Höntzsch D. Die äußere Knochenfixation (Fixateur externe) in der Frakturbehandlung. OP Journal 1997; 13: 20-28
${ }^{7}$ Mooney V, Claudi B. How stable should external fixation be? In: Uhthoff HK (Ed). Current Concepts of External Fixation of Fractures. Berlin, Heidelberg, New York: Springer-Verlag, 1982: 21 -26

8 Pape HC, Krettek C. Surgical priorities in damage control in polytrauma. J Bone Joint Surg [Br] 2003; 85: 478-483

Prof. Dr. med. Dankward Höntzsch Leitender Arzt, Abteilung Medizintechnische Entwicklung

Berufsgenossenschaftliche Unfallklinik

Schnarrenbergstraße 95

72076 Tübingen 